\title{
Visual postural performance after loss of somatosensory and vestibular function
}

\author{
W PAUluS, A STRAUBE, T H BRANDT \\ From the Neurological Clinic, University of Munich, Munich, Federal Republic of Germany
}

SUMMARY Visual stabilisation of body sway in a patient with severe deficits of the vestibular system (due to gentamicin treatment) and the somatosensory system (due to polyneuropathy) was studied. With eyes open the patient was able to stand and walk slowly. With eyes closed he lost balance within one second. In order to optimise visual stabilisation he intuitively searched for nearby visual targets. His postural sway was recorded using posturography. His balance performance deteriorated significantly beyond a distance of $1 \mathrm{~m}$ between the eyes and the surrounding objects and with visual acuity below $\mathbf{0 . 3}$ (experimentally achieved with semitransparent plastic foils). With flicker illumination of decreasing frequencies of the visual surround he needed at least a visual input rate of $17 \mathrm{~Hz}$ in order to maintain an upright body position. The data provide clinical evidence for rapid visuo-spinal control of posture.

Posture and gait control in man is based upon acquisition of preprogrammed patterns of muscle activation initiated and modulated by vestibulovisuo-somatosensory multiloop information about the sensory consequences of self-generated body sway. In normal subjects all three sensory inputs converge and combine to provide redundant information. The particular contribution of visual stabilisation in clinical neurology has long been examined by the Romberg test. ${ }^{1}$ With the aid of posturography the ratio of standing performance with eyes open and eyes closed can be quantified, the so-called Romberg quotient.

Visual stabilisation requires first of all detection of the retinal image shift of the seen environment caused by the head sway. For geometrical reasons the amplitude of this image shift decreases with increasing eyetarget distance. Therefore visual stabilisation is most effective with nearby targets. ${ }^{2}$ Other variables of the

Address for reprint requests: Dr W Paulus, Neurological Clinic, University of Munich, Marchioninistr 15, 8000 Munich 70, Federal Republic of Germany.

Received 30 January 1987 and in revised form 19 April 1987. Accepted I May 1987. visual scene contribute to further complexity of visual compared with vestibular stabilisation. Visual perception of relative body motion depends on the three dimensional structure of the environment as well as on the viewing conditions such as illumination and accommodation. ${ }^{3}$ Factors such as visual acuity, location and size of the stimulus within the visual field, changing eye-target distances and therefore different retinal image velocities are usually involved in varying combinations. ${ }^{2}$

We studied in detail visual stabilisation of posture in a patient whose standing performance strongly depended on visual information after severe damage of labyrinthine function due to gentamicin treatment, and loss of position sense of the feet due to polyneuropathy. He was unable to maintain balance in darkness.

\section{Case report}

In a man aged 35 years the first symptoms of a sensory polyneuropathy developed in parallel with severe diabetes mellitus, which was treated with insulin. He developed diabetic nephropathy with a compensated renal insufficiency and chronic pyelonephritis. Because of severe diabetic small vessel angiopathy two toes were amputated. At the age of 41 
osteomyelitis developed and was treated with gentamicin.

$\mathrm{He}$ was referred to us at the age of 48 years because of standing and walking difficulties in the dark. On admission visual acuity was 1.0 on both eyes. Slight distal paresis due to polyneuropathy was evident in both feet; standing on heels or toes was just possible without support. Ankle and knee jerks could not be elicited. Hypaesthesia below the knees and anaesthesia in both feet was found. Vibration and position sensation were absent in the feet, and significantly affected in both knees and both hands. Hypohidrosis, trophic skin changes, small symmetrical pupils with only a minor reaction to light and frequent diarrhoea suggested an additional autonomic neuropathy.

He was unable to stand or walk in the dark. After eye closure he fell immediately. He used a cane and his gait was slow, careful and broad based. Head movements were avoided; the head was usually slightly bent forward with his eyes searching close visual contact to the floor. If a wall was nearby, he walked along the wall, again searching for close visual targets.

The audiogram revealed a symmetrical hearing loss of $10 \mathrm{~dB}$ at $500 \mathrm{~Hz}$ with slow decline to $30 \mathrm{~dB}$ at $6000 \mathrm{~Hz}$. In the electronystagmogram in darkness a spontaneous nystagmus of $2-3 \%$ to the right was evident. With caloric testing $\left(44^{\circ}\right)$ in darkness on both ears a weak nystagmus response of 3-5 $\%$ angular velocity could be elicited. With sinusoidal chair acceleration $(0.1 \mathrm{~Hz}, V \max 60 \% \mathrm{~s})$ there was no vestibuloocular reflex with the eyes open in darkness.

Peroneal nerve conduction velocities from both legs could not be recorded and there was complete atrophy of the extensor digitorum brevis muscle. Median nerve conduction velocity was reduced to 30 and $31 \mathrm{~m} / \mathrm{s}$. Active denervation and polyphasic action potentials could be found in both opponens pollicis and tibialis anterior muscles.

\section{Posturography}

We studied the influence of eye-target distance, visual acuity and visual input rate on balance performance. The patient was placed on a force-measuring platform with free upright stance in front of a stationary background covered with a random dot pattern of high visual contrast (detailed method in Ref 2). Root mean square values of body sway (RMS) were calculated separately on-line by means of a computer for anterior-posterior (a-p) and right-left (r-l) sway.

Figure (a) depicts the influence of eye-target distance on fore-aft and lateral body sway. On moving from 33 to 50 and $100 \mathrm{~cm}$ eye-background distance only a slight increase in anterior-posterior and right-left sway occurred. With $2 \mathrm{~m}$ distance (two bottom traces) a more pronounced, particularly fore-aft body sway increase, was evident. The increase of body sway at this distance is in good agreement with similar data obtained with posturographic recordings of height vertigo, which also starts at a distance of $2 \mathrm{~m}$ and saturates at about $20 \mathrm{~m}$ distance. ${ }^{45}$ Within this distance range retinal image shift due to normal head sway falls short of the motion discrimination threshold.

In a second experiment visual acuity was experimentally reduced with semi-transparent plastic foils normally used for the better eye in the treatment of amblyopia. ${ }^{2}$ Within the grasping space of $1 \mathrm{~m}$ distance only slightly worse standing performance was measurable with acuities of $1.0,0.6$ and $0 \cdot 3$. Significant unsteadiness occurred with an acuity of $0 \cdot 1$, this, however, only with the high contrast visual back- ground. In a large room with unstructured white walls slight balance support was already needed with an acuity of 0.3 at a distance of $3 \mathrm{~m}$ from the wall.

In a third experiment body sway increased during stroboscopic illumination of the background with decreasing flicker frequencies (fig b, middle), more pronounced for foreaft than for lateral sway. Repeatedly, with $16 \mathrm{~Hz}$ illumination the patient fell down (fig $b$, bottom).

\section{Discussion}

Visual stabilisation of posture differs in several aspects from somatosensory and vestibular stabilisation of body sway. Both somatosensory position sense and vestibular input are invariably interpreted as self-motion perception. The visual system however can transmit two motion sensations: either selfmotion or object motion. Vestibular input in normal subjects seems to serve as a fixed "internal reference"6 and enables a further fine tuning of balance by the visual system. In our patient without appropriate vestibular and somatosensory reference the visual system has both to detect change in size of seen objects and velocity of retinal image shifts and to differentiate between self- and object motion. During voluntary head movements the performance of the visual system itself was further hindered by the absence of sufficient compensatory eye movements (defect vestibuloocular reflex) which causes oscillopsia with increased postural instability.

Severe defects of position sense and vestibular function rarely occur together; in this case they were caused by diabetic polyneuropathy and vestibular damage caused by gentamicin. The main complaint with this combined deficit was a general difficulty with walking, particularly under impaired visual stimulus conditions. The patient's slow and careful standing and walking required conscious movement with the need for serial visual feedback, which normally is provided subconsciously by somatosensory and (in balance regulation) vestibular information.

By means of posturography we were able to confirm the clinical significance of our results obtained in normal subjects concerning the importance of eye-object distance and visual acuity for visual stabilisation of posture. ${ }^{2}$ Without vestibular and somatosensory afferents, otherwise largely redundant visual information proves to be essential for postural balance. Normally with 1 to 4 $\mathrm{Hz}$ flicker illumination (when only changes in position are detected rather than continuous movement) body sway is reduced by $1 / 3$ of the range between eyes open and eyes closed. With $8 \mathrm{~Hz}$ this contribution increases to about $50 \%$ (fore-aft sway) and to $70 \%$ (lateral sway). In normal subjects it saturates at about $16 \mathrm{~Hz}$. The patient with pure visual stabilisation required an input rate of $17 \mathrm{~Hz}$, which is close to the 


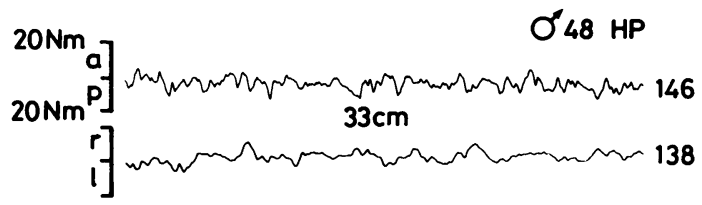

a] pommons 181

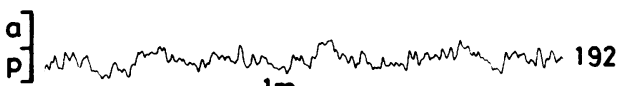

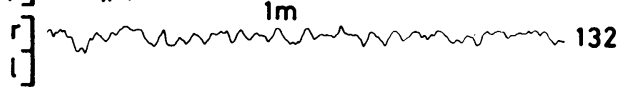

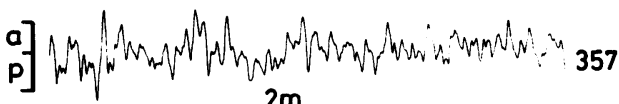

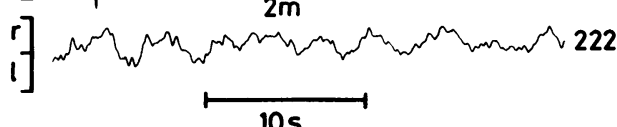

Fig (a)

Fig (a) Differential effects of visual background distances to the eyes (from $33 \mathrm{~cm}$ to $2 \mathrm{~m}$ ) on fore-aft (a-p: upper trace of each pair) and lateral sway ( $r$-l: lower trace of each pair). Original recordings of fore-aft and lateral sway in a patient with almost complete loss of labyrinth function and lower limb joint position sense. Root mean square values of the sway are depicted at the right. Body sway increases with increasing distance between the eyes and the surround especially between 1 and $2 \mathrm{~m}$. (b) Fore-aft and lateral body sway of the same patient under steady room illumination (top) and stroboscopic illumination (distance between eye and visual surround $1 \mathrm{~m}$ ) with different strobe frequencies (18, 17 and $16 \mathrm{~Hz}$ (bottom). Body sway increases with decreasing flicker frequency. With $16 \mathrm{~Hz}$ cannot be maintained for seconds; the patient repeatedly fell from the platform.

input rate which approaches flicker fusion threshold and provides continuous motion perception of the head relative to the visual scene. Impairment of motion perception secondary to reduced flicker frequency may contribute to the observed postural destabilisation. On the other hand this rate of $17 \mathrm{~Hz}$ is in good agreement with experimental findings on rapid visual contribution to motor responses during postural control, which occurs within 100 to $150 \mathrm{~ms}$ for combined postural and visual pertubations. ${ }^{7}$ It complements earlier findings in normal subjects ${ }^{89}$ and patients with cither vestibular or somatosensory lesions ${ }^{9}$ where vision did not appear to be a rapid sensory source for postural reaction in that patients with combined somatosensory and vestibular deficits
$20 \mathrm{Nm}$

$20 \mathrm{Nm}$

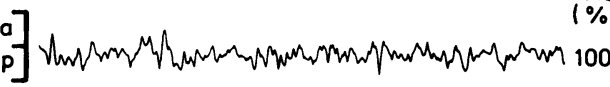

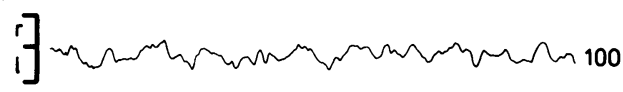

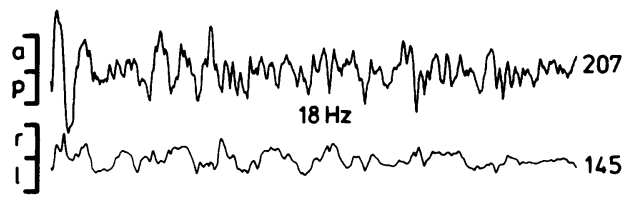
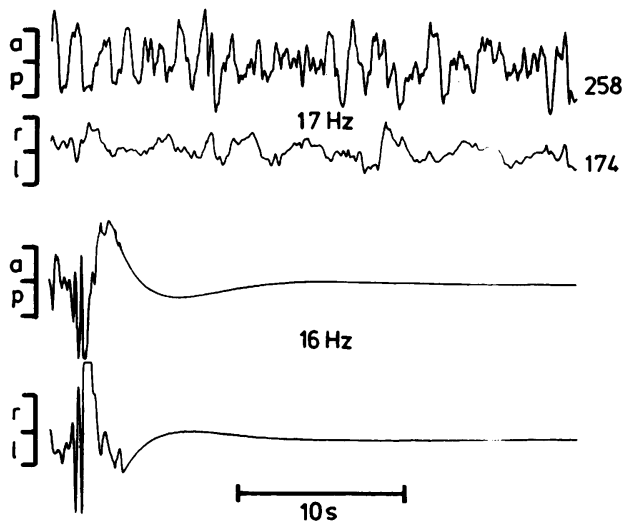

Fig (b)

make use of rapid visual information. The input ratc of $17 \mathrm{~Hz}$ provides a new head position value every $59 \mathrm{~ms}$, which could be used theoretically for a continuous stabilisation of head sway (fecdback sensorimotor loop). Practically, however, we believe in agreement with Droulez and Berthoz, ${ }^{10}$ Nashner, ${ }^{11}$ and Amblard et $\mathrm{al}^{12}$ that the visual system is mainly involved in discontinuous adjustment as a trigger for programmed postural strategies and patterns of muscular activation.

We thank the Alfried Krupp von Bohlen and Halbach foundation and the Deutsche Forschungsgemeinschaft for financial support; Dr D Rushton and $M$ Hawken for critical reading of the manuscript.

\section{References}

I Romberg MH. Lehrbuch der Nervenkrankheiten des Menschen. Berlin: Duncker. 1846.

2 Paulus WM, Straube A, Brandt Th. Visual stabilization of posture. Physiological stimulus characteristics and 
clinical aspects. Brain 1984;107:1143-63.

3 Kapteyn TS, Bles W, Brandt Th, Wist ER. Visual stabilization of posture: effect of light intensity and stroboscopic surround illumination. Agressologie 1979; 20C:191-2.

4 Brandt Th, Arnold F, Bles W, Kapteyn TS. The mechanism of physiological height vertigo. Theoretical approach and psychophysics. Acta Otolaryngol 1980;89:513-23.

5 Bles W, Kapteyn TS, Brandt Th, Arnold F. The mechanism of physiological height vertigo. II. Posturography. Acta Otolaryngol 1980;89:534-40.

6 Nashner LM, Black FO, Wall C. Adaptation to altered support and visual conditions during stance: patients with vestibular deficits. $J$ Neurosci 1982;2:536-44.

7 Nashner LM, Berthoz A. Visual contribution to rapid motor responses during postural control. Brain Res 1978;150:403-7.
8 Vidal PP, Berthoz A, Millanvoye M. Difference between eye closure and visual stabilization in the control of posture in man. Aviat Space Environ Med 1982;53:166-70.

9 Bronstein AM. Suppression of visually evoked postural responses. Exp Brain Res 1986;63:655-8.

10 Droulez J, Berthoz A. Servo-controlled (conservative) versus topological (projective) mode of sensory motor control. In: Bles W, Brandt Th, eds. Disorders of Posture and Gait. Amsterdam: Elsevier 1986:83-98.

11 Nashner LM. Strategies for organization of human posture. In: Igarashi M, Black FO, eds. Vestibular and Visual Control of Posture and Locomotor Equilibrium. Basel: Karger, 1985:1-8.

12 Amblard B, Cremieux J, Marchand AR, Corblanc A. Lateral orientation and stabilisation of human stance: static versus dynamic cues. Exp Brain Res 1985;61:21-37. 